\title{
Limited-capacity identity processing of multiple integers
}

\author{
Dale J. Cohen ${ }^{1}$ - Philip T. Quinlan ${ }^{2}$ \\ Published online: 9 May 2019 \\ (C) The Psychonomic Society, Inc. 2019
}

\begin{abstract}
The architecture of the numerical cognition system is currently not well understood, but at a general level, assumptions are made about two core components: a quantity processor and an identity processor. The quantity processor is concerned with accessing and using the stored magnitude denoted by a given digit, and the identity processor is concerned with recovery of the corresponding digit's identity. BlancGoldhammer and Cohen (Journal of Experimental Psychology: Learning, Memory, and Cognition, 40, 1389-1403, 2014) established that the recovery and use of quantity information operates in an unlimited-capacity fashion. Here we assessed whether the identity processor operates in a similar fashion. We present two experiments that were digit identity variations of Blanc-Goldhammer and Cohen's magnitude estimation paradigm. The data across both experiments reveal a limited-capacity identity processor whose operation reflects cross-talk with the quantity processor. Such findings provide useful evidence that can be used to adjudicate between competing models of the human number-processing system.
\end{abstract}

Keywords Numerical cognition $\cdot$ Numerical architecture $\cdot$ Parallel processing $\cdot$ Capacity limits $\cdot$ Numerical distance effect

Although interest in human numerical cognition has grown considerably over the last 25 years (Cohen Kadosh \& Dowker, 2015), many of the key issues remain underresearched. Here we address two fundamental issues concerning the architecture of the numerical cognition system: (1) whether capacity limits exist on the processes dedicated to the identification of digits, and, (2) whether these processes interact with those dedicated to quantity information (i.e., semantic information). We begin with a brief review of extant models of the architecture of the numberprocessing system, focusing on Arabic digit encoding and identification. We then describe potential configurations of the major components of these models, with particular respect to processing capacity limits. Finally, we present two experiments that adjudicate between these models.

\section{Models of numerical architecture}

McCloskey's abstract-code model (1992; Sokol, McCloskey, Cohen, \& Aliminosa, 1991) is one of the earliest models of the

Dale J. Cohen

cohend@uncw.edu

1 University of North Carolina, Wilmington, NC, USA

2 University of York, York, UK cognitive architecture underlying number processing. The abstract-code model posits separate visual encoding modules for Arabic digits and number words that feed into a single semantic module that stores quantity information in an abstract code. Together, these modules constitute "the number-comprehension system" (McCloskey, Caramazza, \& Basili, 1985). For a multidigit number such as 4,137, the number-comprehension system operates by parsing the number into its constituent digits, retrieving their corresponding quantities, and assigning them to place values. A "number-production system" comprises separate systems for generating responses containing visual digits and number words, respectively. The model is based on a single route from input (characterized by the operation of the numbercomprehension system) to output (characterized by the numberproduction system) via the semantic store of quantity information. Access to semantics is assumed to operate only once a digit has been encoded and identified.

In contrast to this single-route model, various multiple-route models have been considered. For example, arguments have been put forward that posit the existence of an asemantic route as well as the more traditional semantic route (Cohen \& Dehaene, 1991; Deloche \& Seron, 1982). The asemantic route accommodates evidence that some number-processing tasks, such as transcoding, are accomplished in the absence of accessing quantity information (e.g., Deloche \& Seron, 1982).

Such ideas were explored further by Cipolotti (1995), in terms of a dual-route architecture dedicated to processing 
Arabic numerals. The first stage of processing was captured by an Arabic numeral input system and was said to receive "information from early visual processing mechanisms and allows the identification of single digits" (p. 332). The model was heavily influenced by the then-current models of visual word processing (Morton, 1969; Warrington \& Shallice, 1980), whereby letter identity is captured by the recovery of the letter's visual form. Critically, at this stage of processing, neither phonological nor sematic information is accessed; this happens only subsequently, via the operations of the semantic and asemantic routes. Asemantic processing proceeds as a means of recovering spoken forms that then supports spoken number name output. In a later article, Cipolotti and Butterworth (1995) explored a model that posited separate asemantic routes for written Arabic numbers, written named numbers, and spoken numbers. According to Cipolotti (1995), asemantic processing is preferentially activated in order to perform transcoding tasks.

Asemantic processing is also discussed in the context of a variant of the triple-code model of Dehaene (1992). In the variant of this model (see Dehaene \& Akhavein, 1995), object segmentation and feature extraction take place in an initial stage of early visual processing. Subsequent to this are two further modules. One is dedicated to Arabic comprehension and operates to identify visual Arabic digits, regardless of font, size, or color; this seems to be essentially the same as the Arabic numeral input system discussed by Cipolotti (1995). The lexical entries (digit detectors) here are akin to the kinds of letter detectors posited in the interactive activation and competition models discussed by McClelland and Rumelhart (1981) in their account of visual word recognition. The second module is designated verbal comprehension and operates to identify number words, regardless of font, size, or color. This is akin to a lexicon in which the entries are the orthographic forms for number words. The outputs from each of these modules feed forward to the semantic magnitude representation and to a final module, labeled the phonological representation. This module takes the input from the digit and number word lexica and converts these to a spoken form. In allowing interconnections between the two lexica and the phonological representation, the model allows for asemantic transcoding between Arabic and verbal numbers via phonology.

\section{The present theoretical approach}

This early work provided the foundations of much of the numerical cognition research performed today. Here, we examine in detail a central theme of this work: the separation between early visual encoding processes and a later stage of visual identification in which the input stimulus is assigned a digit identity (Cipolotti, 1995; Dehaene \& Akhavein, 1995). We focus solely on the processing of visually presented Arabic digits, in a bid to provide a relatively fine-grained account of how key encoding and identification processes relate to accessing number semantics. As such, this work is akin to that undertaken by researchers in their quest to understand how processes concerning visual featural and letter encoding inform more general theories concerning the mental architecture underpinning word recognition and reading (see, e.g., McClelland \& Rumelhart, 1981; Rumelhart \& Siple, 1974). Although, historically, these "low-level" processes have not featured heavily in traditional accounts, there is now a growing body of evidence that these need to be considered if adequate models of numerical cognition are to be developed (see Cohen, 2009, 2010; Cohen \& Quinlan, 2016; Cohen, Warren, \& Blanc-Goldhammer, 2013; Defever, Sasangie, Vandewaetere, \& Reynvoet, 2012; García-Orza, Perea, Mallouh, \& Carreiras, 2012; Wong \& Szücs, 2013; Zhang, Xin, Feng, Chen, \& Szücs, 2018).

We have chosen to focus on input and decision processes. Figure 1 provides a schematic representation of different accounts of how these input and identification components may be arranged and how they underpin number processing once a digit is presented visually. ${ }^{1}$ Because each component is generally understood in relation to the types of errors it is responsible for, we briefly describe each component with respect to its predicted errors. All of the models assume that the first stage of processing is constituted by visual encoding. The encoding system does not identify or interpret the stimulus, but operates on the physical structure of the stimulus in order to recover shape. As such, the similarity in physical structure between different stimuli is the predicted source of confusions when the encoding stage is operating. Importantly, the specific pattern of these confusions is not task-specific. That is, the physical structure of every digit may be confused with the physical structure of every other digit, and the probability of this confusion is a function of the physical similarity of each digit to every other digit (similar assumptions are central to the interactive activation and competition model of letter processing put forward by McClelland \& Rumelhart, 1981). Cohen and Quinlan (2016) modeled this process in detail. Encoding is characterized as a continuously updating process whereby information is continuously fed to the other processing stages (see Cohen \& Quinlan, 2016).

The other two components are labeled, respectively, the quantity processor and an identify digit processor (henceforth, simply the identity processor). The operations associated with the quantity processor bring about access to the underlying quantity representations linked to each digit. As a consequence, the performance of the quantity processor reflects characteristics of the manner in which quantities are stored and accessed. We align the quantity processor with the types of semantic systems discussed by Cipolotti (1995) and Dehaene and Akhavein (1995), and note that it is sometimes

\footnotetext{
${ }^{1}$ We do not doubt that in certain contexts the recovery of a digit's name is important, such as in digit-naming tasks (see, e.g., Reynvoet, Brysbaert, \& Fias, 2002), but here we limit the discussion to cases that do not necessitate phonological processing. On these grounds, we limit the discussion to putative mechanisms that support visual processing.
} 


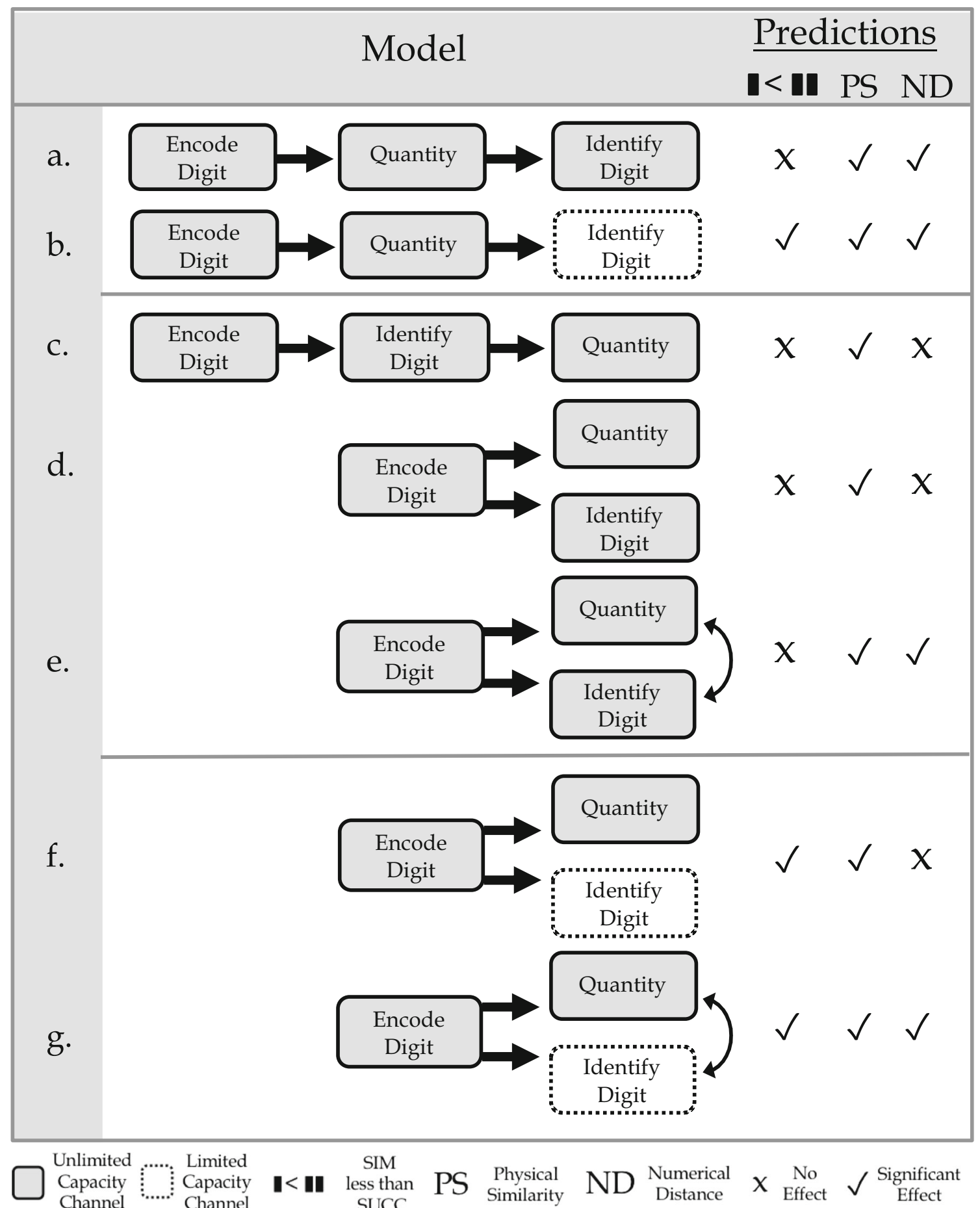

Fig. 1 Schematic representations of the alternative accounts of the human number-processing system considered in the text. PS and ND stand for the experimental variables physical similarity and numerical distance, respectively. A tick in the corresponding column signifies that the

referred to as the approximate number system (Feigenson, Dehaene, \& Spelke, 2004).

The quantity processor operates on the quantity denoted by the symbol, and therefore similarity in denoted quantity is the predicted source of confusions. Furthermore, the specific particular model predicts an effect of the variable, whereas a cross signifies a case in which the model makes no such prediction. The leftmost Predictions column specifies whether or not the model predicts capacity limitations

pattern of these confusions is a function of the quantities being compared. The quantities being compared, in turn, are taskspecific. So, if the task is to determine whether a quantity is greater than or less than a given standard digit such as "5" (or, e.g., if 5 is used as a prime), then the pattern of confusions is 
predicted to be a function of the numerical distance between the presented quantity and 5 (i.e., the comparison quantity). If a different target digit is chosen, then the predictions concerning confusions will change accordingly. The operation of the quantity processor is revealed by confusions regarding numerical distance.

In contrast, the identity processor comprises operations concerned with recovering the corresponding stored identity of a given input digit. ${ }^{2}$ Similar to Cipolotti's (1995) Arabic numeral input system and Dehaene and Akhavein's (1995) "Arabic comprehension," 3 the identity processor is entrained once early visual encoding processes have run their coursefor example, as in the case of taking the digit " 5 " and deriving a unique code (cf. Posner, 1969) that signifies the digit's identity. Such a code underpins being able to make an appropriate discriminative response to the digit. The identity processor operates on the physical structure of the symbol. Therefore, similarity in physical structure is the predicted source of confusions when the identity processor is operating, but now the specific pattern of these confusions is a function of the digits being compared. Again, the identity processor may be activated in a variety of tasks (e.g., identify a numeral, discriminate between numerals, etc.). The digit comparison process is, in turn, a function of the task. For example, if the task is to determine whether a digit is a "5," then the pattern of confusions is predicted to be a function of the physical similarity between the presented digit and "5" (i.e., the comparison digit). If a different target digit is chosen, then the predictions concerning structural confusions will change accordingly. In this way, the operation of the identity processor is revealed by these kinds of physical similarity relations. Finally, the operation of the identity processor is disambiguated from the operation of the encoding system because the confusions of the encoding system are common to all tasks (i.e., the presented digit is confused with all other digits), whereas the confusions of the identity processor are task-specific (i.e., the presented digit is confused with the comparison digit).

It is important to note that the identify processor is tuned to differentiate digits from one another, rather than being a generalized shape categorizer. We hypothesized this because

\footnotetext{
${ }^{2}$ In discussing the identity processor, we are limiting ourselves to discussion of the visual processes that underpin the ability to link the visual structure of a digit with its visual identity - for example, the ability to identify the input digit for five as " 5 ." We assume that there are analogous processes in the other senses, so that, for instance, an utterance can be identified as the spoken form /fiv/. We are agnostic as to whether the corresponding spoken form of a visual digit is automatically accessed when the visual digit is presented (though see Damian, 2004), but we do assume that processing phonological forms plays no obligatory role in visual identification. The empirical consequences of this assumption, however, were not tested here. Nonetheless, we did test the statistical significance of a model of processing that takes into account the physical similarity of the input digits defined in visual terms.

${ }^{3}$ We are agnostic as to whether the identify processor completes tasks beyond recovering the corresponding stored identity of a given input digit (e.g., multidigit operations, parity, etc., as claimed by Dehaene, 1992).
}

Cohen (2009) demonstrated that the physical similarity of digits is a function of the symbol structure, rather than of visual form. Cohen's (2009) physical similarity function first simplifies the digits into the eight-line structure incorporated in a digital clock. Then a ratio is calculated of the number of lines that the two digits share, divided by the number of lines they do not share that are required to make up the two digits. Because the physical similarity function of digits is derived from the underlying structure of the digits, it predicts errors regardless of the font in which a digit is displayed (see Cohen, 2009). A basic assumption of our approach is that there are principled differences between early visual encoding and digit identification. Such differences are made explicit in the models of Cipolotti (1995) and Dehaene and Akhavein (1995), and analogical differences have been explored most thoroughly in the context of visual letter processing (see, e.g., Coltheart, 1972). Furthermore, there is neurophysiological evidence of the separation between encoding and digit identification (e.g., Pinel et al., 1999).

In distinguishing between the identity and quantity processors, we acknowledge that the respective processes are separable. We also note, at the outset, that the successful operation of these processors is predicated on the successful encoding of the input digit. In a recent article, Cohen and Quinlan (2016) provided a detailed model and computer simulations of the encoding and quantity comparison processes. Cohen and Quinlan showed, via computer simulations, that it is computationally feasible for quantity information to be accessed from a numerical symbol without this being dependent on accessing the identity of that symbol. Nevertheless, Cohen and Quinlan were agnostic as to how and when symbol identification occurs. In the following section, we broaden the discussion to include the three components shown in Fig. 1, and we are particularly interested in how digits are identified. We explore the processing capacity of the identity processor and the degree to which the identity and quantity processors are functionally independent of one another. In the following, we sketch out various possible cognitive architectures that we take to be plausible structures for basic number processing and our primary intention is to adjudicate between these. Figure 1 provides schematic representations of the particular architectures that we will consider in detail.

\section{The present empirical approach}

We start by addressing the issue of processing capacity limits. In this respect, a seminal reference is Shiffrin and Gardner (1972), who were interested in the degree to which stimulus identification (qua letter identification) is limited in capacity. In their paradigm, performance was examined across two conditions: a simultaneous condition (here termed SIM) and a successive condition (here termed SUCC). In the SIM condition, participants were presented with a brief display 
containing four characters, which were masked upon removal. In the SUCC condition, participants were briefly presented with two displays in succession - each containing two characters, which were masked upon removal. In both the SIM and SUCC conditions, the character displays were presented for the same brief amount of time (i.e., $50 \mathrm{~ms}$ ). In this way, the amount of time available to extract information from a twocharacter display was the same as that available to extract information from a four-character display. On the assumption that character identification is a limited-capacity process, it was predicted that participants would be more accurate in the SUCC than in the SIM condition. Critically, however, the results showed that there was, essentially, no difference in accuracy across the two conditions: Consequently, Shiffrin and Gardner concluded that, at least in the conditions tested, letter identification is unlimited in capacity. In summarizing the relevant findings and the ensuing literature, Pashler (1998, p. 123) concluded that when a small number of characters have to be processed, there are no capacity limitations. This is tempered by the fact that evidence of capacity limitations only emerges once the difficulty of target discrimination is considerable (see, e.g., Kleiss \& Lane, 1986). Furthermore, after a careful review of the relevant word-processing literature, Lachter, Forster, and Ruthruff (2004) argued strongly that there is no identification without attention, with the implication that stimulus identification is capacity-limited (see Pashler, 1998, chap. 5).

In an effort to better understand the architecture of the numerical cognition system, Blanc-Goldhammer and Cohen (2014) addressed whether the quantity processor is capacitylimited. Blanc-Goldhammer and Cohen used a pared-down version of the Shiffrin and Gardner (1972) task. All of the characters were digits, and the task was to identify the diagonal on which the largest digit occurred. The rationale was the same as before: Namely, if the act of deriving relative quantity information is capacity-limited, then performance ought to be better in the SUCC than in the SIM condition. Contrary to this prediction, however, a key finding was that there was no overall difference in performance across these two conditions. Blanc-Goldhammer and Cohen concluded that the process of comparing the quantities conveyed by integer digits is unlimited in capacity.

Figure 1 presents alternative models of the numerical cognition system. To aid in interpreting the figure, a gray box with a solid border indicates unlimited-capacity processing, whereas a white box with a dashed border indicates limited-capacity processing. Figure 1 assumes that all processing stages prior to the quantity processor occur in an unlimited-capacity fashion. This assumption is necessary because, in a stage model, a limited-capacity processor acts as a bottleneck. This bottleneck reduces the information flow to the later stages, causing the later stages to mimic limited-capacity processing as well (regardless of the later stages' processing capacity). Given that
Blanc-Goldhammer and Cohen (2014) found no evidence of limited-capacity processing in their task, in all models in Fig. 1 we assume that the quantity processor operates with unlimited capacity. Furthermore, given that encoding operates prior to accessing quantities, we conclude that encoding also operates in an unlimited-capacity fashion. Finally, we rule out the possibility of a limited-capacity identity processor that operates prior to the unlimited-capacity quantity processor. With these limitations, Fig. 1 outlines the remaining models that are possible. Figures $1 a$ and $1 b$ set out sequential systems, wherein the operations related to the quantity processor precede the operations of the identity processor. In Fig. $1 a$, the identity processor is unlimited in capacity, whereas in Fig. 1b, the identity processor is limited in capacity.

Immediately, however, we can rule out all models in which the quantity processor is located before the identity processor (as in Figs. $1 a$ and $b$ ). Such models predict that quantity must be processed before digit identification can take place. If this were the case, digit identification would be influenced by the same factors that influence quantity comparison. Evidence of accessing quantity information exists when participants' reaction times (RTs) for correct responses decrease monotonically as the numerical distance between the two digits increases (e.g., Moyer \& Landauer, 1967). This is termed the numerical distance effect and is taken to indicate the operation of the quantity processor (Banks \& Flora, 1977; Besner \& Coltheart, 1979; Dehaene \& Akhavein, 1995; E. M. Duncan \& McFarland, 1980; Schwartz \& Heinze, 1998).

Critically, Cohen (2009) demonstrated that digit identification can take place without activation of the quantity processor (for additional supporting evidence, see Ratinckx, Brysbaert, \& Fias, 2005, and Pansky \& Algom, 2002). Cohen (2009) conducted a digit identification task in which, on every trial, a single visual digit was presented, and the participant simply had to identify whether the digit was a "5." The primary interest was performance on "different trials," in which a digit other than " 5 " was presented. If performance was driven primarily by digits accessing their corresponding quantity representations, then the RTs on the different trials should scale according to the numerical distance between the presented digit and 5. In contrast, the performance on different trials revealed the importance of physical similarity. That is, RTs varied according to how visually similar the actual presented digit was to the digit " 5 " (termed a physical similarity effect). Here we assume that physical similarity effects reflect, in part, operations concerned with digit identification (see also Cohen et al., 2013). As a consequence, we assert that when participants engage in a digit identification task, performance will, in large measure, reflect effects of physical similarity that are key to that particular identification judgment.

Having ruled out Models $1 a$ and $1 b$, different accounts need to be considered. Figures $1 c-g$ show various architectures based on the premise that the quantity processor operates 
in an unlimited-capacity fashion, but the individual cases differ in other critical regards. For instance, Fig. $1 c$ shows the case in which the two processors operate sequentially, with the identity processor situated before the quantity processor. This model captures key features of the models discussed by McCloskey (1992), Cipolotti (1995), and Dehaene and Akhavein (1995). Following visual encoding, a digit is first identified, and then semantics are accessed. In the particular model shown, the quantity processor is of unlimited capacity (see Blanc-Goldhammer \& Cohen, 2014). The identity processor is also unlimited in capacity, and therefore it does not create a bottleneck prior to the unlimited-capacity quantity processor. If it is assumed that both processors must run to completion prior to a response, then we can rule out this model for the same reasons that Models $1 a$ and $1 b$ have been ruled out: Performance in simple digit identification tasks does not reveal effects of numerical distance (see Cohen, 2009). The reasoning is that if a response can only be emitted once the corresponding quantity has been accessed, then effects of numerical distance should be obtained. However, if a response can be initiated once the identity processor has completed, then Model $1 c$ remains viable.

Models $1 d-1 g$ are radically different from the sequential accounts because, in all cases, the two processors are seen to operate in parallel. Models $1 d$ and $1 e$ assume that both processors operate in an unlimited-capacity way. Whereas the processors in Model $1 d$ are functionally independent, in Model $1 e$ cross-talk is possible between the processors. Model 1e allows for information exchange across the processors (see Mordkoff \& Yantis, 1991). According to one reading of the model, the suggestion is that the processors function in a mutually dependent way, but this form of dependency has already been put in question by the data that show that digit identification can proceed independently of mechanisms concerning the accessing of quantity information. A more flexible reading is that cross-talk between the processors is possible but not necessary. Further discussion of cross-talk will be included as the material unfolds. The final models, shown in the Figs. $1 \mathrm{f}$ and $1 \mathrm{~g}$, are similar to Models $1 d$ and $1 e$, respectively, with the exception that Models $1 f$ and $1 g$ are based on the assumption that the identity processor operates in a limited-capacity way.

To adjudicate among the viable alternative accounts in Fig. 1, we modified the Blanc-Goldhammer and Cohen (2014) paradigm so that the task was simply to decide whether a single target digit (a "5") was presented. Our primary concern was whether the identity processor is limited in capacity, as would be revealed by the finding that performance was better in the SUCC condition than in the SIM condition, or, unlimited in capacity, as supported by the finding of equivalent levels of performance in the two conditions. Models $1 c-1 e$ assume that the identity processor is unlimited in capacity, and Models $1 f$ and $1 g$ assume that the identity processor is limited in capacity.

In the rightmost columns in Fig. 1, we map out what each model predicts in terms of the physical similarity function and the numerical distance function. A detailed description of the physical similarity of the digits used can be found in Cohen (2009), and numerical distance standardly refers to the degree to which the quantities referenced by the digits differ. As we described above, we assume that the physical similarity function (here, the task-specific physical similarity to the digit "5") will be observed when the identity processor is activated, and the numerical distance function (here, the task-specific numerical similarity to the quantity " 5 ") will be observed when the quantity processor is activated. Because we assume that the identity processor is necessary to complete the identification task, we should always observe the physical similarity function in the data. The numerical distance function should be observed only when the quantity processor is active. This would happen if the quantity processing is a prerequisite to the identity process or if the two processes interact. Therefore, Models $1 c, 1 d$, and $1 f$ all predict an effect of physical similarity and not of numerical distance. Models $1 e$ and $1 g$ predict effects of both physical similarity and numerical distance. Not considered here, in any detail, are models in which digit identity can be completed solely through the quantity processor. These models would predict an effect of numerical distance but not physical similarity, and given the emerging evidence against this pattern, this outcome is taken to be extremely unlikely and not considered further (Cohen, 2009; Cohen et al., 2013; Defever et al., 2012; García-Orza et al., 2012; Wong \& Szücs, 2013; Zhang et al., 2018).

Because the present task necessitates activation of the identity processor, all models predict effects of physical similarity. However, only a subset of the models predict effects of numerical distance. On these grounds, any putative numerical distance effect will be key in adjudicating between the different models. Moreover, the most revealing finding would be that numerical distance effects emerged only after the influence of physical similarity had been removed from the data. As Cohen (2009) has shown, physical similarity and numerical distance are positively correlated, and typically effects of numerical distance may be abolished once effects of physical similarity have been accounted for.

\section{Experiment 1}

\section{Method}

Participants A total of 122 naïve participants volunteered for class credit. All participants were undergraduates at the University of North Carolina at Wilmington (UNCW). Although it would have been preferable to record 
demographic data, at UNCW the students' average age is 22 , there are $62 \%$ females, and $83 \%$ of the students are white, $6 \%$ are African American, $6 \%$ are of one or more other races, and $4 \%$ are unknown. About $7 \%$ of the students are Hispanic.

To determine the sample size, we started with Kleiss and Lane (1986), who had conducted a similar experiment with letters, from which we estimated effect sizes ranging from about $d=0.5$ to $d=1.3$ (depending on the physical similarity of the letters). However, we tempered this with the conclusion of Brysbaert and Stevens (2018) that "it is bad practice to use effect sizes of published article [sic] as an estimate for power analysis, because they tend to be exaggerated. Much better is to assume effect sizes of $d=.4$ or $d=.3$ (the typical effect sizes in psychology)" (p. 16). With this in mind, for the key analysis (a paired-samples $t$ test) we assumed a conservative $d=0.3$, so that for power to equal .8 the required $\mathrm{n}$ is 93 . We therefore set the minimum number of participants to 100 .

Apparatus and stimuli All stimuli were presented on a 24-in. LED color monitor with a $72-\mathrm{Hz}$ refresh rate controlled by a Macintosh Mini running OS X. The resolution of the monitor was $1,920 \times 1,200$ pixels.

Figure 2 provides a schematic representation of the sequence of events in trials in the SIM and SUCC conditions. (To be clear, the figure incorporates all conditions across the two experiments, and in Exp. 1 the SUCC condition is labeled SUCC $\mid 0$.) Prior to each trial, four digits were randomly chosen (with replacement) from the digits " 1 " to " 9 ," excluding "5." On half the trials, the target (i.e., " 5 ") randomly replaced one of the four distractor digits. The characters were presented as Arabic digits in Arial font, subtending about $0.68^{\circ}$ of visual angle vertically.

Procedure and design The display timings were as shown in Fig. 2. In all major respects, the methods were the same as those used by Blanc-Goldhammer and Cohen (2014). However, in this case the participants were instructed that, on each trial they were to determine whether the target (i.e., "5") was present in the display. A single target was used, to reduce any interference that might result from switching targets between trials. Furthermore, a target of " 5 " is optimal, because it allows for the greatest number of single-digit distractors that are symmetrically distributed above and below the target. Half of the participants were instructed to press $k$ if the target (i.e., " 5 ") was present, and $d$ if it was not. The other half of the participants used the reverse key assignment. To facilitate task familiarity, displays were presented for $75 \mathrm{~ms}$ in the practice trials; on experimental trials, this was reduced to $66 \mathrm{~ms}$.

Within a given testing session, the SIM and SUCC conditions were blocked. Each block contained 75 trials. The presentation conditions alternated, for a total of four blocks. Twelve practice trials were presented prior to the start of each of the first two blocks. The participants were not told that there were different presentation conditions in the different blocks. Half of the participants were presented the SUCC condition first, and the other half were presented the SIM condition first. Participants were allowed to take self-timed breaks between blocks.

\section{Results and discussion}

Thirteen of the participants experienced computer problems, and their data were removed prior to analysis. We calculated $d^{\prime}$ for each of the remaining participants for each condition $(M=1.29, S D=$ 0.56 ), and we removed participants who had a $d^{\prime}<0.2$ (at or near chance) in at least one condition. We applied this exclusion criterion because chance performance indicated that the participant was hitting floor in that condition. If a participant hits floor in at least one condition, the difference between the SIM and SUCC conditions would be biased low for that individual. This would shift the overall results toward identifying no difference between the SIM and SUCC conditions. The unlimited-capacity model predicts equal sensitivities in these conditions, and to include data from chance performers in the eventual dataset would compromise our ability to offer a reasonable test of this prediction. This criterion excluded three participants (average performance after removing the three participants: $M=1.32, S D=0.54){ }^{4}$

A paired $t$ test revealed that participants were more sensitive to the target in the SUCC condition $(M=1.37, S D=0.495)$ than in the SIM condition $(M=1.27, S D=0.577), t(105)=2.15, p=.03$. This result suggests that identifying a specific digit is a capacitylimited process. Clearly, the effect is small, $d=0.2$; therefore, we designed Experiment 2 in a bid to set the effect on more a more solid basis.

We also assessed the influences of physical similarity and numerical distance on performance in the nontarget trials. Clear predictions can be made regarding the relations between false alarms (i.e., errors on nontarget trials) and physical similarity, and, respectively, numerical distance. Accordingly, participants should be more inaccurate as the physical similarity between the nontargets and the target increases, whereas they should be less inaccurate as the numerical distance between the nontargets and the target increases. In both cases, the participant would presumably be misperceiving the nontarget as the target. If processing capacity is limited, the identities of some but not all of the digits in a trial would be processed. A default assumption is that sampling of the digits would be random; hence, over trials the expected interference from the nontarget digits would be indexed by the average physical similarity and numerical distance of those digits. To test these predictions, we calculated the average physical similarity and average numerical distance for each four-digit nontarget combination. We calculated these averages by doing the following:

1. For each digit in a four-digit nontarget combination, we calculated the physical similarity relative to the digit "5"

\footnotetext{
${ }^{4}$ The patterns of results remained the same with the no $d^{\prime}$ exclusion criteria.
} 


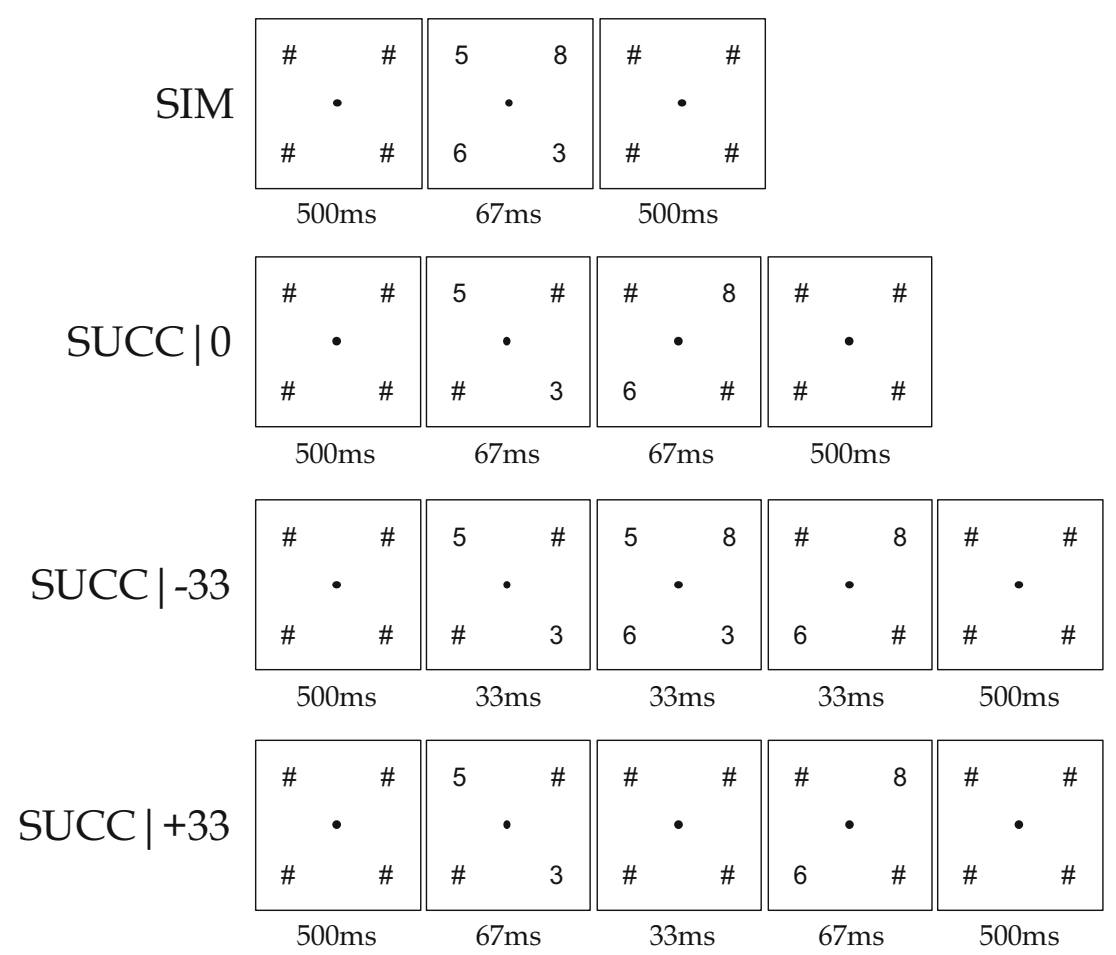

Fig. 2 Schematic representation of the key trial events for the experimental conditions used in the experiments. Each row in the figure corresponds to a trial, with time running from left to right. The SIM and SUCC | 0 conditions were common to both experiments. All four

(see Cohen, 2009, for a complete description of the physical similarity measures).

2. We then averaged across these four physical similarities to get the average physical similarity for that specific fourdigit nontarget combination.

3. We repeated this process for each unique, four-digit nontarget combination.

4. To get the average numerical distance, we repeated Steps $1-3$, substituting the calculation of numerical distance for physical similarity.

At this point, each unique, four-digit nontarget combination was summarized by its average physical similarity and average numerical distance relative to the target " 5 ." The average physical similarity and average numerical distance of these four-digit nontarget combinations were correlated: SIM $r=-.41$, SUCC $r=-.38$. To obtain stable estimated false alarm rates, we collapsed trials across (a) participants and (b) the four-digit nontarget combinations with the same average physical similarity statistic and the same average numerical distance statistic (e.g., the data for all the four-digit nontarget combinations in which the average $\mathrm{PS}=3$ and average $\mathrm{ND}=2$ would be combined, collapsing over participants). These false alarm rates were used as the dependent variable in the regression models described below. Figure 3 presents these data for the SIM and SUCC conditions. conditions were tested in Experiment 2 only. The \# indicates a mask, as described in the text. Although in all the SUCC cases the left diagonal is shown as being presented before the right diagonal, the order of presentation of these diagonals was randomized
All the models in Fig. 1 assume that the physical nature of the digits influences performance in the tasks. ${ }^{5}$ Therefore, showing an effect of physical similarity on performance was of no consequence in adjudicating between the different models under consideration. What is germane is whether, having taken account of the effects of physical similarity, effects of numerical distance would still emerge. To test this line of reasoning, we adopted the following analytic approach. To assess the influence of numerical distance after removing the influence of physical similarity, we calculated two linear models for each condition. First, we computed a linear regression with false alarm rate (described above) as the criterion variable and physical similarity as the predictor variable. We then extracted the residuals of this model and used them as the criterion in the second model, in which numerical distance was the predictor variable. If the regression coefficient of the second model were statistically significant, we might conclude that there was an influence of numerical distance after removal of the influence of physical similarity. As such, our analysis was designed to disambiguate the models, rather than to disambiguate the shared variance.

For the SIM condition, physical similarity alone accounted best for the data, $r^{2}=.15, F(1,195)=35.48, p<.001$, slope $=$

\footnotetext{
${ }^{5}$ The physical structure of the numerical symbol must be encoded, at least in part, in order to provide meaningful information to the quantity process (see Cohen \& Quinlan, 2016).
} 


\section{Simultaneous}

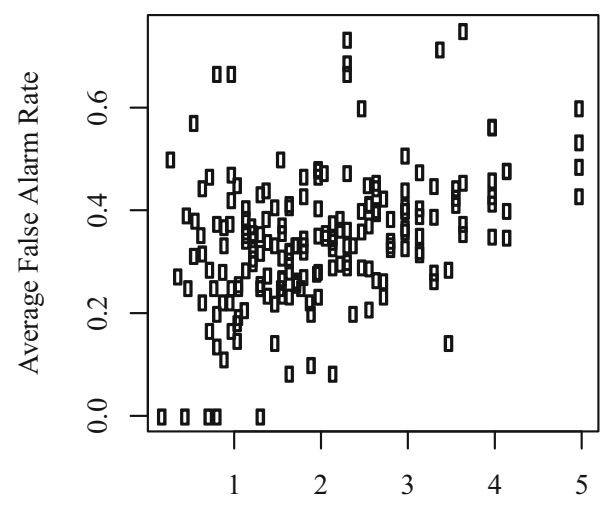

Average Physical Similarity

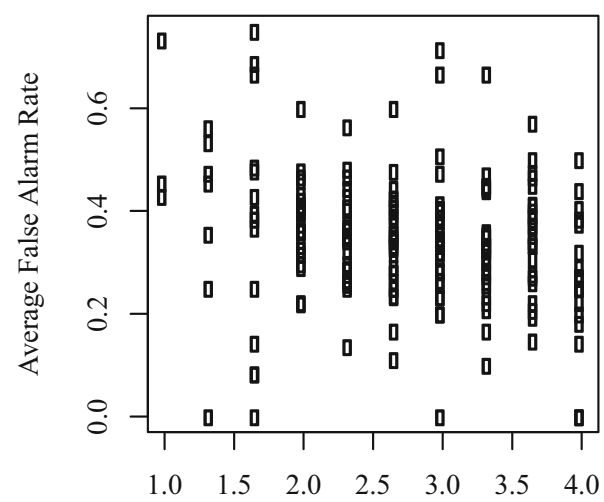

Average Numerical Distance

Fig. 3 Experiment 1's false alarm rates, as a function of physical similarity (top) and numerical distance (bottom) for the SIM (left) and SUCC (right) conditions. These are the data used in the regression

0.05 . Numerical distance added no predictive benefit, $F(1$, $195)=0.68$, n.s. These results reveal that as the physical similarity of the display digits to the target increased, the participants' false alarms increased.

For the SUCC condition, both physical similarity, $r^{2}=.07$, $F(1,200)=15.97, p<.001$, slope $=0.03$, and numerical distance, $r^{2}=.04, F(1,200)=7.42, p<.001$, slope $=-$ 0.03 , were significant predictors. These results show that as the physical similarity of the other display digits to the target increased, the participants' false alarms increased. In addition, as the numerical distance of the other display digits from the target increased, the participants' false alarms also decreased.

In sum, the observed differences in sensitivity between the sequential and simultaneous conditions support the conclusion that digit identification is a capacity-limited process. Moreover, from the more detailed analyses of the data, both physical similarity and numerical distance affected performance. Following on from the previous work by Cohen

\section{Successive}


analysis, with each four-digit display having a PS-ND value. For each PS-ND combination, we averaged false alarm rates over participants

(2009), the effect of physical similarity was as predicted, given that visual encoding plays a critical role in completing the task. Performance, in part, reflected the degree to which the different digits could be distinguished from the digit "5." In addition, performance (in the SUCC task, at least) was also modulated by the numerical distance between the target and the nontarget digits in nontarget displays. This finding shows that numerical quantities were being accessed, despite the fact that successful responding did not necessitate this.

Overall, therefore, the data sit most comfortably with Model $1 g$. The evidence is consistent with assuming the operation of a limited-capacity identity processor together with cross-talk between the quantity and identity processors. Nonetheless, the numerical distance effect is surprising. In both the Cohen (2009) study and the present case, digit identification was key, and yet, whereas in the earlier study no effect of numerical distance was found, here such an effect did emerge. 
To examine this contrasting pattern of findings, we carried out a second experiment. Now, the testing conditions were adapted so that display exposure was manipulated in a particular and systematic way. The standard SUCC condition (i.e., SUCC | 0) was repeated, and two further versions of the SUCC condition were added. In the SUCC $\mid-33$ condition, the presentation of the second pair of characters began $33 \mathrm{~ms}$ before the removal of the first pair. The first pair of characters was presented for $33 \mathrm{~ms}$, then all four characters were presented for $33 \mathrm{~ms}$, and then the first pair of characters, on one diagonal, were removed, leaving only the second-diagonal characters visible for $33 \mathrm{~ms}$. In the SUCC $\mid+$ 33 condition, a masker display was interpolated between the two-character displays for $33 \mathrm{~ms}$. That is, there was an interstimulus interval (ISI) of $33 \mathrm{~ms}$ between the removal of the first pair of characters, on one diagonal, and presentation of the second pair, on the other diagonal. All four masks remained visible during this ISI.

The conditions were motivated by the following reasoning. The intention behind the SUCC $\mid-33$ condition was to attempt to overload the system by introducing the intermediate display between the two two-item displays. Here the system had to process fewer stimuli per millisecond than in the SIM condition, but more stimuli per millisecond than in the SUCC $\mid 0$ condition. In contrast, the intervening masking display in the SUCC $\mid+33$ condition was intended to allow additional time for the system first to process the initial pair of digits prior to the onset of the second pair. Here the system had to process fewer stimuli per millisecond than in the SUCC $\mid 0$ condition. If processing capacity is unlimited, then the results should show $\mathrm{SIM}=\mathrm{SUCC} \mid-33=$ SUCC $\mid 0=$ SUCC $\mid+33$. If, however, processing capacity is limited, as was suggested by the results of Experiment 1, we would expect performance to be poorer in the SIM condition than in the SUCC conditions, $\mathrm{SIM}<(\mathrm{SUCC} \mid-33 \&$ SUCC $\mid 0 \&$ SUCC $\mid+$ $33)$. More subtly, varying the timing of the successive condition provided a way to assess the severity of the capacity limits. If capacity limits are large relative to the information being processed, then every incremental addition of time would provide a performance advantage: SIM $<$ SUCC $\mid-33<$ SUCC $\mid 0<$ SUCC $\mid$ +33 . If, however, the processing capacity limits are small relative to the information being processed, then only the first incremental addition of time would provide a performance advantage: $\mathrm{SIM}<$ SUCC $\mid-33=$ SUCC $\mid 0=$ SUCC $\mid+33$. This would be the case because the additional time increment in the SUCC $\mid-33$ condition would nullify the relatively small influence of processing capacity limits. Because the influence of processing capacity limits was already nullified, the additional time increments in the SUCC $\mid 0$ and SUCC $\mid+33$ conditions could not produce further performance increases.

Finally, assuming that the sample mean and standard deviation equaled the population mean and standard deviation, respectively, a post-hoc power analysis revealed that Experiment 1 had a power of .57. We therefore increased the number of participants in order to increase the power of the experiment. The increased power would help determine whether the influence of numerical distance in the SIM condition was undetected because it was not present or because it was simply too small, given the power of the first experiment.

\section{Experiment 2}

\section{Method}

Participants A total of 229 naïve participants (from the same university sample) volunteered for class credit. The sample size was determined in the same way as in Experiment 1. The key analysis of Experiment 2 was with respect to a mixed-effects model, because of the increased power that this model provides. A priori power analysis for a mixed-effects model, however, is a relatively uncertain process (Brysbaert \& Stevens, 2018). Therefore, we calculated power assuming a paired $t$ test, with the knowledge that the mixed-effects model analysis would provide greater power than that estimated for the $t$ test. The power analysis, assuming an effect size equal to that obtained in Experiment 1, indicated a required $n$ of 182. We set the minimum number of participants to 200 in order to ensure enough power to identify effects, if they existed.

Apparatus and stimuli The apparatus and stimuli in Experiment 2 were identical to those of Experiment 1.

Procedure and design The general procedure in Experiment 2 was the same as that in Experiment 1. However, now the four conditions-SIM, SUCC $\mid 0$, SUCC $\mid-33$, and SUCC $\mid+33$ were administered in separate blocks of trials. Each block contained 146 trials. The order of the presentation conditions was randomized over four blocks. Twelve practice trials were presented prior to the start of each block. The participants were simply told to identify the target number " 5 " and were not told that there were different presentation conditions. Participants were allowed to take self-timed breaks between blocks.

\section{Results and discussion}

Sixteen participants experienced computer problems and were removed from the dataset prior to the analysis. As in Experiment 1, we calculated $d^{\prime}$ for each of the remaining participants for each condition $(M=1.35, S D=0.66)$. Eighteen participants were removed who had a $d^{\prime}<0.2$ (at or near chance) in at least one condition (performance after removing the 18 participants: $M=1.44, S D=0.61){ }^{6}$

A mixed-model analysis of variance, with participants as a random factor and the presentation condition as a fixed factor, revealed a significant effect of presentation condition, $F(3$,

\footnotetext{
${ }^{6}$ The patterns of results remained the same with no $d^{\prime}$ exclusion criteria.
} 
$582)=83.3, p<.001$. Calculating effect sizes for mixedeffects models is not straightforward, but Nakagawa and Schielzeth (2013) have shown that useful information can be gained by calculating the marginal and condition $r^{2}$ s of these models $\left(r_{\text {marginal }}^{2}=.1, r_{\text {conditional }}^{2}=.70\right)$. A post-hoc power analysis using the simr package in $\mathrm{R}$ revealed power $=1.0$ (95\% confidence intervals: .98-1.0). A Tukey's HSD test revealed that the participants were less sensitive to the target in the SIM condition than in the three SUCC conditions $(p<$ .001 ; see Table 1). The three SUCC conditions were not significantly different from one another $(p>.05)$. This result replicated the central finding of Experiment 1: Digit identification is a capacity-limited process.

We again assessed the influences of physical similarity and numerical distance on performance in the same manner as in Experiment 1 . Because participants were equally sensitive to the three SUCC conditions, we combined the data for these conditions in this analysis. Again, physical similarity and numerical distance were correlated in the four-digit displays: $\operatorname{SIM} r=-$ .39 , combined SUCC $r=-.38$. Figure 4 presents false alarms plotted as a function of physical similarity and numerical distance for the SIM and combined SUCC conditions. For the SIM condition, both physical similarity, $r^{2}=.09, F(1,201)=19.38, p<$ .001 , slope $=0.03$, and numerical distance, $r^{2}=.08, F(1,201)=$ $17.69, p<.001$, slope $=-0.03$, were significant predictors. For the combined data for the SUCC conditions, both physical similarity, $r^{2}=.13, F(1,609)=86.9, p<.001$, slope $=0.03$, and numerical distance, $r^{2}=.02, F(1,609)=86.9, p<.001$, slope $=-$ 0.02 , were also significant predictors. As in Experiment 1, these results reveal that as the physical similarity of the digits to the target increased, the participants' false alarms increased. In addition, as the size of the numerical distance of the digits from the target increased, the participants' false alarms decreased. These final analyses established that both physical similarity and numerical distance influenced performance in the same way as was shown in Experiment 1. More particularly, the initially unpredicted effect of numerical distance found in the SUCC condition in Experiment 1 was found in the data for both the SIM and SUCC conditions in Experiment 2. Thus, the lack of an effect of numerical distance in the SIM condition in Experiment 1 was likely the result of a small effect that required increased power in order to be detected. In sum, the data fit most comfortably with the parallel, interactive-processors account shown in Fig. $1 g$. Responses revealed effects of both physical similarity

Table 1 Mean $d^{\prime}, S D$, and $n$ for each condition of Experiment 2

\begin{tabular}{llll}
\hline Condition & Mean & $S D$ & $n$ \\
\hline SIM & 1.11 & 0.50 & 195 \\
SUCC $\mid-33$ & 1.57 & 0.56 & 195 \\
SUCC $\mid 0$ & 1.50 & 0.61 & 195 \\
SUCC $\mid+33$ & 1.57 & 0.65 & 195 \\
\hline
\end{tabular}

and, to a lesser extent, numerical distance, and we therefore concluded that the evidence is more in line with Model $1 g$ than with Model $1 f$.

\section{General discussion}

The central findings, replicated across two experiments, support the parallel interactive processors account of how the human number system operates (i.e., as is shown in Fig. $1 g$ ). The present data show that digit identification is a limitedcapacity process. In addition, effects of both physical similarity and numerical distance are present in the data. The effects of numerical distance, though small, were found in three for the four general cases tested (with the exception of the SIM data for Exp. 1), and these will be considered in more detail shortly.

The primary finding of the present research is that the digit identity processor has a limited capacity. This conclusion is supported by the data from both Experiments 1 and 2, which showed that participants were more accurate in the SUCC conditions than in the SIM condition. Furthermore, Experiment 2 showed that participants were equally sensitive in all three SUCC conditions. This finding suggests that the limited capacity is small relative to the amount of information being processed. That is, although the addition of a small increment of time in the SUCC $\mid-33$ condition improved performance over that of the SIM condition, additional increments of time in the SUCC $\mid 0$ and SUCC $\mid+33$ conditions provided no increase in sensitivity. This suggests that capacity was not the critical constraint limiting performance in the SUCC $\mid 0$ and SUCC $\mid+33$ conditions. Therefore, the digit identity processor has a limited capacity, but that limit is not severe relative to the amount of information being processed.

The finding that the digit identity processor has a limited capacity is consistent with Lachter et al. (2004), who claimed that there is no identification without attention. Nevertheless, a limited-capacity digit identity processor may be considered an unintuitive finding. This is because Blanc-Goldhammer and Cohen (2014) determined that the quantity processor has unlimited capacity. The data suggest that within the numerical cognition system, the processes concerning digit identity do not precede those concerned with the extraction of quantity. More likely, these separate processors operate in parallel.

The evidence that the identity and quantity processors are functionally separate provides some insight into how quantities are represented by numerical symbols. BlancGoldhammer and Cohen (2014) demonstrated that, in a field of four numerical symbols, participants could locate in parallel the symbol that denoted the greatest quantity. However, the present experiment demonstrated that people could not identify those same symbols concurrently. This suggests that extracting a quantity from a numerical symbol has some 


\section{Simultaneous}

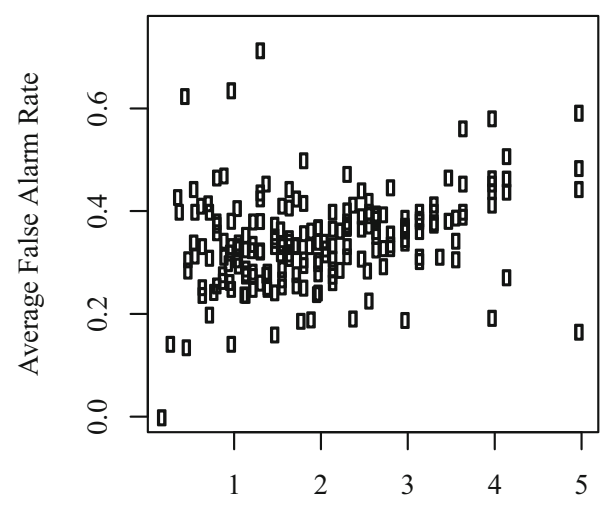

Average Physical Similarity

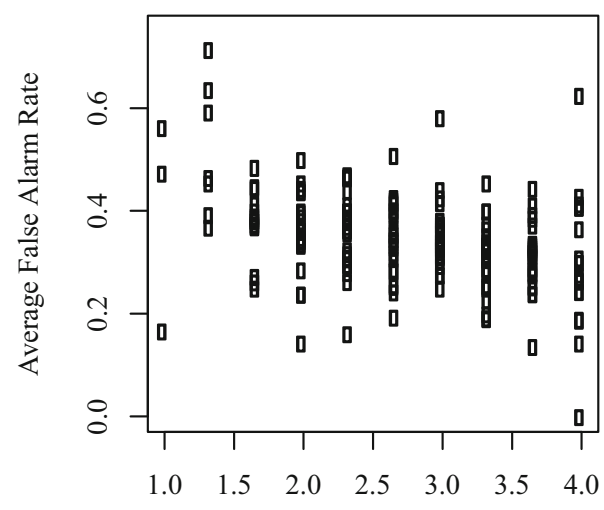

Average Numerical Distance

Fig. 4 Experiment 2's false alarm rates as a function of physical similarity (top) and numerical distance (bottom) for the SIM (left) and combined SUCC (right) conditions. These are the data used in the regression

similarity to extracting a quantity from, say, a field of dots. This is because one can judge which of two fields of dots has a greater quantity without knowing the exact identity of the quantity associated each field of dots (see, e.g., Burgess \& Barlow, 1983). So, numerical symbols, when processed for quantity rather than identity, appear to activate a noisy representation of quantity (see Cohen \& Quinlan, 2016) and no precise identity information. In contrast, when numerical symbols are processed for precise identity rather than quantity, they appear to sometimes activate identity information and no quantity information (see Cohen, 2009), and at other times activate both identity and quantity information (Exps. 1 and 2). Below we discuss this inconsistency further.

Effects of physical similarity were present in the data for both the SIM and SUCC conditions in both experiments. Moreover, these effects were expressed consistently across all cases. Participants were less accurate when processing displays that contained digits that were highly physically similar to " 5 " than
Successive

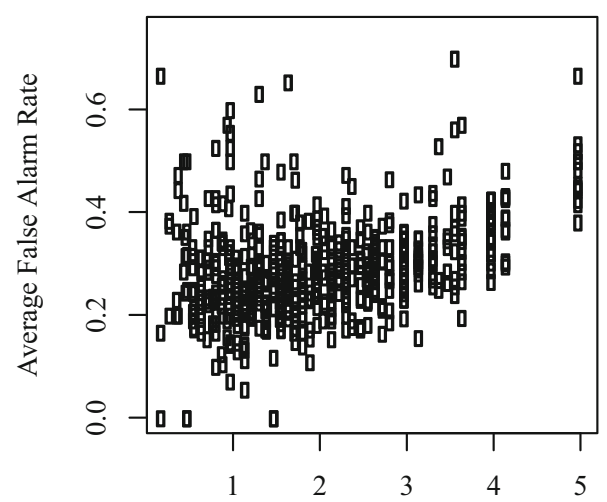

Average Physical Similarity

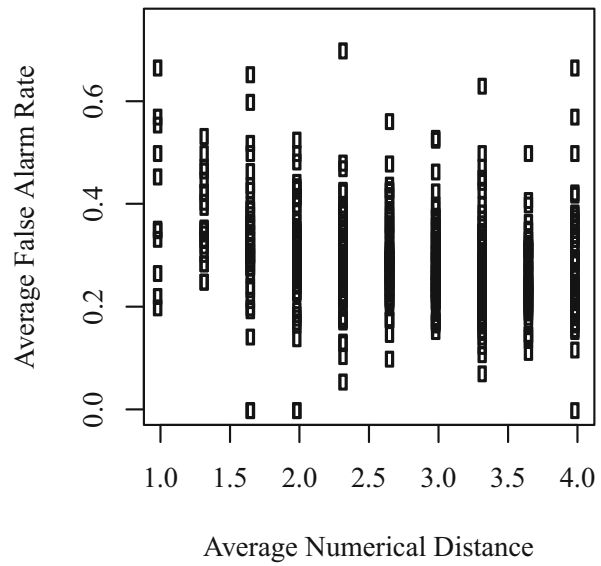

analysis, with each four-digit display having a PS-ND value. For each PS-ND combination, we averaged false alarm rates over participants

when the digits were highly physically dissimilar to "5." This pattern of responding fits comfortably with the notion that digit identification reflects processes concerning the physical nature of the digits, and more particularly that errors arise due to similarity relations between the input digits and the target digit. Different errors are assumed to arise when the identification task is changed accordingly. The nature of the identification processes is, nonetheless, seen to be capacity-limited in nature and to be distinct from that concerning the recovery of quantity information. This account applies equally to the case in which a single digit is presented in each trial (i.e., as in Cohen, 2009) and here, where four digits were presented and the same target digit was always probed.

Participants were also less accurate in their responses on target-absent trials when the nontarget digits were numerically close to 5 than when the digits were numerically distant from 5. This pattern of performance arose in all cases except in the SIM condition in Experiment 1. Nevertheless, the increased power in Experiment 2 likely made it possible to detect the 
small effect of numerical distance in the SIM condition that had been difficult to detect in Experiment 1. Such effects are as predicted, if it is assumed that digit identification relies on quantity representations being accessed, but this was not a necessary precursor to successful task completion: The task was essentially simple pattern classification that did not necessitate accessing any semantic information. The general pattern of findings leads to the conclusion that the identity and quantity processors exhibit functional cross-talk. Critically, the fact that the data revealed effects of both physical similarity and numerical distance shifts the focus away from Model $1 f$ onto Model $1 g$. Model $1 f$ suggests that a response could be based on the operation of either processor, with no influence of the other, but the data show that this is not so. Responses revealed the influence of both processors, and therefore the evidence is more in line with Model $1 g$ than with Model $1 f$.

We take such cross-talk to be a desirable feature (cf. Mordkoff \& Yantis, 1991). That is, if one is presented with a visual quantity (e.g., dots), it is possible to understand quantity and to verbally label the corresponding number (e.g., say "fiv"). Similarly, if one is presented with the sounds "fiv," it is possible to access the corresponding quantity demanded by a mental arithmetic problem. However, cases are beginning to emerge in which task performance does not depend on such cross-talk (Cohen, 2009; García-Orza et al., 2012; Zhang et al., 2018), as well as cases in which the operation of a processor is revealed even though successful task completion does not demand it (as in the experiments here). Such demonstrations should be considered against other cases in which effects are clearly context-dependent and emerge only as a consequence of the task constraints (see, e.g., Campbell, 2011; Cohen \& Dehaene, 1995). Clearly, future work needs to address such contrasting cases, with a view to better understanding which functional components are influential in a given task, and why. Nonetheless, the context effects that do occur as a consequence of particular task constraints can perhaps best be understood by allowing flexible cross-talk between the quantity and identity processors.

Although Model $1 \mathrm{~g}$ can accommodate the present data, we may ask why effects of numerical distance arose here, when similar effects have not been present in other digit identity tasks when physical similarity was taken into account Cohen (2009; see also García-Orza et al., 2012, and Zhang et al., 2018). This appears surprising, given that digit identification was the basic task in Cohen's (2009) earlier study, as it was here. Nonetheless, at the level of method, the two cases are strikingly different. In the earlier study, on every trial a single digit was presented until response, whereas here, on every trial four digits were presented very briefly and were patternmasked. As a consequence, both the timing constraints and the number of stimuli present on a trial differed across the two cases. Either or both of these might be crucial in understanding the contrasting patterns of performance in the two cases.
We simply take it that the different paradigms challenge the number-processing system in different ways.

In the present model, we make the following stipulations:

1. The identity processor operates in a limited-capacity fashion. This is evidenced by the results of Experiments 1 and 2.

2. The identity processor is most efficient when only a single imperative stimulus is present (Cohen, 2009). This is evidenced by the decrements in performance that are observed in cases in which more than one target is present (see, e.g., J. Duncan, 1980).

3. Recovery of digit identity from the identity processor is more accurate than recovery of digit identity from the quantity processor. Because the quantity processor recovers noisy quantity representations (e.g., Cohen \& Quinlan, 2016), any identity information recovered from the quantity processor will also be noisy.

4. Recovery of digit identity from the quantity processor is faster for multiple digits (as in Blanc-Goldhammer \& Cohen, 2014) than is recovery of digit identity from the identity processor for multiple digits. This is because the quantity processor has unlimited capacity, whereas the identity processor has limited capacity.

When identifying a digit, the identity processor is preferred because of Point 3 above. Furthermore, on single-stimulus trials in which the task is to identify the digit, performance reflects the efficient operation of the identity processor. However, in cases in which more than one imperative stimulus is presented and the displays are brief, the different stimuli compete for access to the identity processor. Competition for the identity processor acts to impede it. Here, the unlimitedcapacity quantity processor might have influenced performance by processing some of the digits for identification. When this occurs, accuracy would decrease, and the numerical distance effect would be present.

We note, in accepting the interactive model proposed in Fig. $1 g$, that this model stands in contrast to the conclusions regarding processing drawn by Dehaene (1996). Dehaene (1996) reported performance in a number comparison task in which, on every trial, the participant made a speeded judgment as to whether the quantity conveyed by the stimulus was greater or less than 5. The stimulus was either a number name (e.g., FOUR) or an Arabic digit. Dehaene (1996) discussed the work in the context of a variant of the triple-code model based on a sequential-stage account of processing. Stage 1 is labeled "identification," Stage 2 is "comparison," and Stage 3 is "response." Adopting an additive-factors logic (Sternberg, 1975), Dehaene (1996) argued that the notation of the stimulus would tap into the identification component, the signified quantity of the stimulus would tap into the comparison component, and the response side would tap into the response component. 
Generally speaking, the results revealed patterns of additivity across these different variables, suggesting that the different components are also additive and not interactive in their operations. Clearly this contrasts with our conclusion that stimulus identification and the accessing of semantics can operate in an interactive way.

There are several reasons why the Dehaene (1996) results do not directly contradict those reported here. First, the paradigms in the respective cases were very different. Here the multidigit arrays were presented briefly and masked, whereas in Dehaene's (1996) study a single stimulus was presented briefly but was unmasked. As was discussed above, we suggest that the interactions between the identification stage and the quantity stage are only manifest when the identification stage is overwhelmed (as occurred here but not in the case reported by Dehaene, 1996). Second, Dehaene's (1996) sequential-stage account assumed that the stimulus must be "identified" prior to accessing quantity. Without this assumption, the additive-factors logic is not valid. We believe that identification is not necessary for quantity to be accessed. Rather, the shape of the digit (or word) alone can activate stored quantity information (see Cohen \& Quinlan, 2016). As such, Dehaene (1996) may not be an adequate test of independence between the identity and quantity processors. In this regard, the patterns of additivity reported by Dehaene (1996) are tangential to the effects we report.

\section{Limitations}

Finally, we note some limitations of the present research. First, the predicted physical similarity effect and numerical distance effect are inherently correlated $(r \sim .4)$. Because one cannot statistically disambiguate the shared variance, we analyzed the data consistent with the theoretical testing of models. That is, all of our models predicted a physical similarity effect. Therefore, the critical feature for disambiguating the models was whether a numerical distance effect was present after removal of the physical similarity effect. Our analyses were directed at answering the following question: "Is numerical distance necessary to explain some of the data, or can all be explained parsimoniously with the physical similarity effect alone?" It is, nonetheless, possible that future models might be proposed that lead to alternative accounts, but at this stage, speculating about what these might be is not warranted or helpful.

Second, some of the statistically reliable effects of numerical distance reported here were small. For instance, the effect of numerical distance in the SUCC condition in Experiment 2 accounted for only $2 \%$ of the total variance. Indeed, in no case did the effect of numerical distance exceed $8 \%$ of the total variance. As in any such case, there is the question of whether very small effects are of any psychological significance (see, e.g., Prentice \& Miller, 1992). We have chosen to take the effects as being of some theoretical import, although others may choose not to be convinced of this. We did so because the very small effects reflect the influence of a key variable that has been taken by many to be the critical factor that determines performance in simple numerical tasks (see, e.g., Dehaene \& Akhavein, 1995).

\section{Conclusions}

We have made some progress in mapping out the basic number-processing architecture. We claim that the parallel interactive account shown in Fig. $1 g$ currently provides the best account of performance across a range of simple numerical cognition tasks. Following encoding, separate quantity and identity processors concurrently operate, respectively, to recover a digit's stored quantity representation and to generate a structural code that supports the digit's conventional identity. The present data were interpreted as showing that these processors operate in parallel in an interactive fashion. Whereas accessing quantity information operates rapidly and effortlessly, digit identification is a more effortful endeavor that reflects interpretive operations that depend on the visual decoding of digits. Whereas certain first-order quantity comparisons can be carried out with unlimited capacity, digit identification is capacity-limited.

Author note The data for all experiments are deposited at UNC Dataverse: https://dataverse.unc.edu/dataverse/limitedCapacity2019. Neither of the experiments was preregistered.

\section{References}

Banks, W. P., \& Flora, J. (1977). Semantic and perceptual processes in symbolic comparisons. Journal of Experimental Psychology: Human Learning and Memory, 3, 278-290. https://doi.org/10. 1037/0096-1523.3.2.278

Besner, D., \& Coltheart, M. (1979). Ideographic and alphabetic processing in skilled reading of English. Neuropsychologia, 19, 467-472. https://doi.org/10.1016/0028-3932(79)90053-8

Blanc-Goldhammer, D., \& Cohen, D. J. (2014). Unlimited capacity parallel quantity comparisons of multiple integers. Journal of Experimental Psychology: Learning, Memory, and Cognition, 40, 1389-1403. https://doi.org/10.1037/a0036843

Brysbaert, M., \& Stevens, M. (2018). Power analysis and effect size in mixed effects models: A tutorial. Journal of Cognition, 1, 1-20. https://doi.org/10.5334/joc. 10

Burgess, A., \& Barlow, H. B. (1983). The precision of numerosity discrimination in arrays of random dots. Vision Research, 23, 811-820. https://doi.org/10.1016/0042-6989(83)90204-3

Campbell, J. I. D. (2011). Semantic priming with product verification but not production. Canadian Journal of Experimental Psychology, 65, 254-258. https://doi.org/10.1037/a0024250

Cipolotti, L. (1995). Multiple routes for reading words, why not numbers? Evidence from a case of Arabic numeral dyslexia. Cognitive Neuropsychology, 12, 313-342. https://doi.org/10.1080/ 02643299508252001 
Cipolotti, L., \& Butterworth, B. (1995). Towards a multiroute model of number processing: Impaired number transcoding with preserved calculation skills. Journal of Experimental Psychology: General, 124, 375-390. https://doi.org/10.1037/0096-3445.124.4.375

Cohen, D. J. (2009). Integers do not automatically activate their quantity representation. Psychonomic Bulletin \& Review, 16, 332-336. https://doi.org/10.3758/PBR.16.2.332

Cohen, D. J. (2010). Evidence for direct retrieval of relative quantity information in a quantity judgment task: Decimals, integers, and the role of physical similarity. Journal of Experimental Psychology: Learning, Memory, and Cognition, 36, 1389-1398. https://doi.org/10.1037/a0020212

Cohen, D. J., \& Quinlan, P. T. (2016). How numbers mean: Comparing random walk models of numerical cognition varying both encoding processes and underlying quantity representations. Cognitive Psychology, 91, 63-81. https://doi.org/10.1016/j.cogpsych.2016. 10.002

Cohen, D. J., Warren, E., \& Blanc-Goldhammer, D. (2013). Cross-format physical similarity effects and their implications for the numerical cognition architecture. Cognitive Psychology, 66, 355-379. https:// doi.org/10.1016/j.cogpsych.2013.03.001

Cohen Kadosh, R., \& Dowker, A. (Eds.). (2015). The Oxford handbook of numerical cognition. Oxford, UK: Oxford University Press.

Cohen, L., \& Dehaene, S. (1991). Neglect dyslexia for numbers? A case report. Cognitive Neuropsychology, 8, 39-58. https://doi.org/10. 1080/02643299108253366

Cohen, L., \& Dehaene, S. (1995). Number processing in pure alexia: The effect of hemispheric asymmetries and task demands. Neurocase, 1 , 121-137. https://doi.org/10.1080/13554799508402356

Coltheart, M. (1972). Visual information processing. In P. C. Dodwell (Ed.), New horizons in psychology 2 (pp. 62-85). Harmondsworth, UK: Penguin.

Damian, M. F. (2004). Asymmetries in the processing of Arabic digits and number words. Memory \& Cognition, 32, 164-171. https://doi. org/10.3758/BF03195829

Deloche, G. \& Seron, X. (1982). From one to 1: An analysis of a transcoding process by means of neuropsychological data. Cognition, 12, 119-149. https://doi.org/10.1016/0010-0277(82) 90009-9.

Defever, E., Sasangie, D., Vandewaetere, M., \& Reynvoet, B. (2012). What can the same-different task tell us about the development of magnitude representations? Acta Psychologica, 140, 35-42. https:// doi.org/10.1016/j.actpsy.2012.02.005

Dehaene, S. (1992). Varieties of numerical abilities. Cognition, 44, 1-42. https://doi.org/10.1016/0010-0277(92)90049-N

Dehaene S. (1996). The organization of brain activations in number comparison: Event-related potentials and the additive-factors method. Journal of Cognitive Neuroscience, 8, 47-68. https://doi.org/10. 1162/jocn.1996.8.1.47

Dehaene, S., \& Akhavein, R. (1995). Attention, automaticity, and levels of representation in number processing. Journal of Experimental Psychology: Learning, Memory, and Cognition, 21, 314-326. https://doi.org/10.1037/0278-7393.21.2.314

Duncan, E. M., \& McFarland, C. E. (1980). Isolating the effects of symbolic distance, and semantic congruity in comparative judgments: An additive-factors analysis. Memory \& Cognition, 8, 612-622. https://doi.org/10.3758/BF03213781

Duncan, J. (1980). The locus of interference in the perception of simultaneous stimuli. Psychological Review, 87, 272-300. https://doi.org/ 10.1037/0033-295X.87.3.272

Feigenson, F., Dehaene, S., \& Spelke, E. (2004). Core systems of number. Trends in Cognitive Sciences, 8, 307-314. https://doi.org/10.1016/j. tics.2004.05.002

García-Orza, J., Perea, M., Mallouh, R. A., \& Carreiras, M. (2012). Physical similarity (and not quantity representation) drives perceptual comparison of numbers: Evidence from two Indian notations.
Psychonomic Bulletin \& Review, 19, 294-300. https://doi.org/10. 3758/s13423-011-0212-8

Kleiss, J. A., \& Lane, D. M. (1986). Locus and persistence of capacity limitations in visual information processing. Journal of Experimental Psychology: Human Perception and Performance, 12, 200-210. https://doi.org/10.1037/0096-1523.12.2.200

Lachter, J., Forster, K. I., \& Ruthruff, E. (2004). Forty-five years after Broadbent (1958): Still no identification without attention. Psychological Review, 111, 880-913. https://doi.org/10.1037/0033295X.111.4.880

McClelland, J. L., \& Rumelhart, D. E. (1981). An interactive activation model of context effects in letter perception. Psychological Review, 88, 375-407. https://doi.org/10.1037/0033-295X.88.5.375

McCloskey, M. (1992). Cognitive mechanisms in numerical processing: Evidence from acquired dyscalculia. Cognition, 44, 107-157. https://doi.org/10.1016/0010-0277(92)90052-j

McCloskey, M., Caramazza, A., \& Basili, A. (1985). Cognitive mechanisms in number processing and calculation: Evidence from dyscalculia. Brain and Cognition, 4, 171-196. https://doi.org/10. 1016/0278-2626(85)90069-7

Mordkoff, J. T., \& Yantis, S. (1991). An interactive race model of divided attention. Journal of Experimental Psychology: Human Perception and Performance, 17, 520-538. https://doi.org/10.1037/0096-1523. 17.2.520

Morton, J. (1969). The interaction of information in word recognition. Psychological Review, 76, 165-178. https://doi.org/10.1037/ h0027366

Moyer, R. S., \& Landauer, T. K. (1967). Time required for judgements of numerical inequality. Nature, 215, 1519-1520. https://doi.org/10. $1038 / 2151519 \mathrm{a} 0$

Nakagawa, S., \& Schielzeth, H. (2013). A general and simple method for obtaining R2 from generalized linear mixed-effects models. Methods of Ecology and Evolution, 4, 133-142. https://doi.org/10. 1111/j.2041-210x.2012.00261.x

Pansky, A., \& Algom, D. (2002). Comparative judgment of numerosity and numerical magnitude: Attention preempts automaticity. Journal of Experimental Psychology: Learning, Memory, and Cognition, 28, 259-274. https://doi.org/10.1037//0278-7393.28.2.259

Pashler, H. E. (1998). The psychology of attention. Cambridge, MA: MIT Press.

Pinel, P., Le Clec'H, G., Van de Moortele, P. F., Naccache, L., Le Bihan, D., \& Dehaene, S. (1999). Event-related fMRI analysis of the cerebral circuit for number comparison. NeuroReport, 10, 1473-1479. https://doi.org/10.1097/00001756-199905140-00015

Posner, M. I. (1969). Abstraction and the process of recognition. In G. H. Bower \& J. T. Spence (Eds.), The psychology of learning and motivation (Vol. 3, pp. 43-100). New York: NY: Academic.

Prentice, D. A., \& Miller, D. T. (1992). When small effects are impressive. Psychological Bulletin, 112, 160-164. https://doi.org/10.1037/ 0033-2909.112.1.160

Ratinckx, E., Brysbaert, M., \& Fias, W. (2005). Naming two-digit Arabic numerals, evidence from masked priming studies. Journal of Experimental Psychology: Human Perception and Performance, 31, 1150-1163. https://doi.org/10.1037/0096-1523.31.5.1150

Reynvoet, B., Brysbaert, M., \& Fias, W. (2002). Semantic priming in number naming. Quarterly Journal of Experimental Psychology, 55A, 1127-1139. https://doi.org/10.1080/02724980244000116

Rumelhart, D. E., \& Siple, P. (1974). Process of recognizing tachistoscpically presented words Psychological Review, 81, 99118. https://doi.org/10.1037/h0036117

Schwartz, W., \& Heinze, H. J. (1998). On the interaction of numerical and size information in digit comparison: A behavioral and event-related potential study. Neuropsychologia, 36, 1167-1179. https://doi.org/ 10.1016/S0028-3932(98)00001-3 
Shiffrin, R. M., \& Gardner, G. T. (1972). Visual processing capacity and attentional control. Journal of Experimental Psychology, 93, 72-82. https://doi.org/10.1037/h0032453

Sokol, S. M., McCloskey, M., Cohen, N. J., \& Aliminosa, D. (1991). Cognitive representations and processes: Inferences from the performance of brain-damaged subjects Journal of Experimental Psychology: Learning, Memory, and Cognition, 17, 355-376. https://doi.org/10.1037/0278-7393.17.3.355

Sternberg, S. (1975). Memory scanning: New findings and current controversies. Quarterly Journal of Experimental Psychology, 27, 1-32. https://doi.org/10.1080/14640747508400459

Warrington, E. K., \& Shallice, T. (1980). Word form dyslexia. Brain, 103, 99-112. https://doi.org/10.1093/brain/103.1.99

Wong, B., \& Szücs, D. (2013). Single-digit Arabic numbers do not automatically activate magnitude representations in adults or in children:
Evidence from the symbolic same-different task. Acta Psychologica, 144, 488-498. https://doi.org/10.1016/j.actpsy.2013. 08.006

Zhang, L., Xin, Z., Feng, T., Chen, Y., \& Szücs, D. (2018). Physical similarity or numerical representation counts in same-different numerical comparison, physical comparison and priming tasks? Quarterly Journal of Experimental Psychology, 71, 670-687. https://doi.org/10.1080/17470218.2016.1276944

Publisher's note Springer Nature remains neutral with regard to jurisdictional claims in published maps and institutional affiliations. 